\title{
Pneumocystis jirovecii pneumonia induced by low-dose methotrexate in a patient with chronic urticaria*
}

\author{
Sheng-Huei Wang ${ }^{1}$ \\ Yu-Huei $\mathrm{Li}^{2}$ \\ Chan-Yuan Chang ${ }^{4}$
}

\author{
Shih-En Tang ${ }^{1}$ \\ Kuang-Yu Wei ${ }^{3}$
}

DOI: http:/ / dx.doi.org/10.1590/abd1806-4841.20175392

\begin{abstract}
Methotrexate has immunosuppressive effects and is administered for refractory chronic urticaria. We present a case of Pneumocystis jirovecii pneumonia in a patient with refractory chronic urticaria managed by low-dose weekly methotrexate treatment (total cumulative dose $195 \mathrm{mg}$ ). Our study highlights the importance of providing prompt diagnosis and treatment of Pneumocystis jirovecii pneumonia in patients with chronic urticaria under methotrexate therapy.
\end{abstract}

Keywords: Methotrexate; Pneumonia, Progressive interstitial, of sheep; Urticaria

\section{INTRODUCTION}

Chronic urticaria, characterized by continuous or intermittent urticarial eruption of wheals or hives for more than six weeks, has a $\sim 0.5 \%$ to $1 \%$ lifetime prevalence in the general population. ${ }^{1-2}$ Antihistamines are the mainstay treatment and can control chronic urticaria in the majority of patients. Methotrexate (MTX) is an alternative agent with immunomodulatory effects that is employed in refractory chronic urticaria. However, the adverse effects or complications of MTX therapy in patients with chronic urticaria are scarcely reported. We present a rare case of Pneumocystis jirovecii pneumonia in a patient with refractory chronic urticaria receiving weekly MTX treatment. Our report highlights the importance of providing prompt diagnosis and treatment of Pneumocystis pneumonia in chronic urticaria patients receiving MTX therapy.

\section{CASE REPORT}

The patient, a 42-year-old woman, presented to our emergency department with a two-week history of dry cough, a oneweek history of progressive dyspnea and chest distress when taking a deep breath, and a four-day history of fever. She had an outpatient medical visit three days prior to presentation without symptom improvement. Of significance was her three-year history of chronic spontaneous urticaria on the face and forehead, which was initially controlled with first or second generation antihistamine agents. This was not associated with autoimmunity, as there was no evi- dence of functional autoantibodies or other clinical manifestations. However, prednisolone (10-30g/day) had been added during the previous year due to disease progression; MTX with folic acid supplementation had been employed during the previous three months as follows: $7.5 \mathrm{mg} /$ week for two weeks, $10 \mathrm{mg} /$ week for six weeks, and $15 \mathrm{mg}$ / week for eight weeks (total dose, 195mg). In addition, the patient had a history of allergic rhinitis and bronchial asthma.

On examination, temperature was $38.6^{\circ} \mathrm{C}$, pulse was 120 beats/min, respiratory rate was 20 breaths/min, and blood pressure was 130/74 mmHg. Cardiopulmonary auscultation revealed inspiratory and expiratory fine crackles in both lung bases. Chest radiography showed increased infiltrations in the bilateral perihilar region and lower lung zones (Figure 1). Blood analysis revealed a leukocyte count of 7,130 cells/mL (88.6\% polymorphonuclear neutrophils, $3.6 \%$ lymphocytes) and a C-reactive protein level of $6.99 \mathrm{mg} / \mathrm{dL}$ (reference, $0.8 \mathrm{mg} / \mathrm{dL}$ ). Chest computed tomography demonstrated diffuse ill-defined ground-glass opacities, tiny nodular and centrilobular opacity, and interstitial thickening in the bilateral upper and lower lung (Figure 2).

The patient was transferred to the intensive care unit due to the development of impending respiratory failure. Results of polymerase chain reaction-based analysis of sputum specimens were positive for Pneumocystis jirovecii and negative for human immunodeficiency virus. Cotrimoxazole $(20 \mathrm{mg} / \mathrm{kg})$ in three divided doses

Received on 18.11.2015

Approved by the Advisory Board and accepted for publication on 15.02.2016

* Study carried out at the Tri-Service General Hospital, National Defense Medical Center - Taipei, Taiwan.

Financial support: none.

Conflict of interest: none.

1 Division of Pulmonary and Critical Care Medicine, Department of Internal Medicine, Tri-Service General Hospital, National Defense Medical Center - Taipei, Taiwan, Republic of China.

2 Division of Endocrinology and Metabolism, Department of Internal Medicine, Tri-Service General Hospital, National Defense Medical Center - Taipei, Taiwan, Republic of China.

Division of Nephrology, Department of Internal Medicine, Tri-Service General Hospital, National Defense Medical Center - Taipei, Taiwan, Republic of China. 4 Division of Infectious Diseases and Tropical Medicine, Department of Internal Medicine, Tri-Service General Hospital, National Defense Medical Center Taipei, Taiwan, Republic of China.

C 2017 by Anais Brasileiros de Dermatologia 


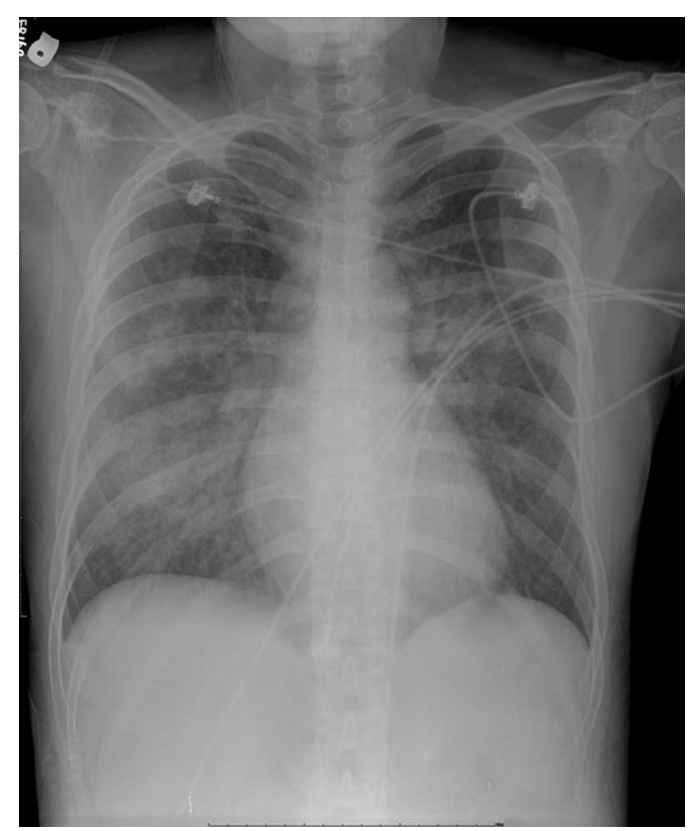

Figure 1: Chest radiography showed increased infiltrations in the bilateral perihilar region and lower lung zones

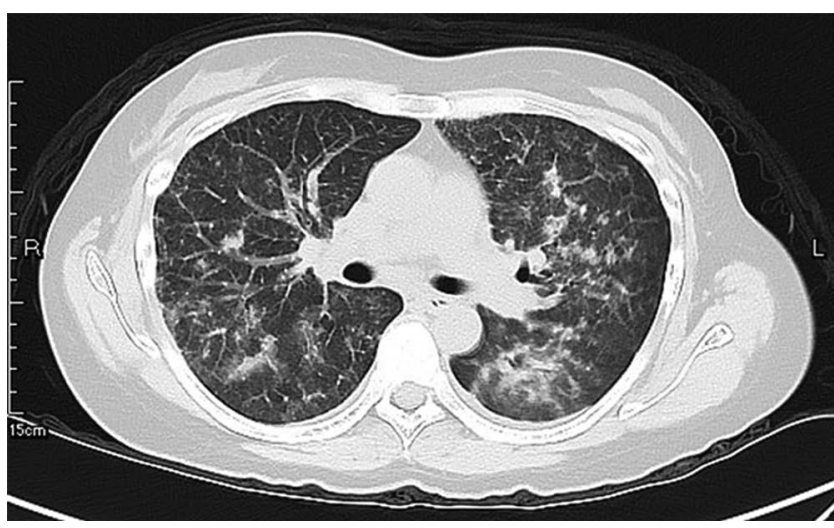

FIGURE 2: Chest computed tomography demonstrated diffuse ill-defined ground-glass opacities, tiny nodular and centrilobular opacity, and interstitial thickening in the bilateral upper and lower lung

and 40mg of methylprednisolone every 12 hours were added to the therapeutic regimen. The patient was discharged with complete recovery 10 days later.

\section{DISCUSSION}

The recommended first-line treatment for patients with chronic urticaria consists of new-generation, non-sedating $\mathrm{H}_{1}$-antihistamines. However, $40 \%$ to $55 \%$ of patients who have a poor response to antihistamines, even with maximal doses, require additional second-line treatments such as systemic corticosteroids, cyclosporine, MTX and omalizumab. ${ }^{3}$
MTX is one of the immunomodulatory agents employed in refractory chronic urticaria, with a recommended weekly mean dose of $15 \mathrm{mg} .{ }^{1}$ The pharmacological mechanism of MTX in chronic urticaria is inconclusive, with a shortage of literature on the subject. Gach et al. reported the effects of MTX on neutrophil adhesion and leukotriene synthesis, whereas Perez et al. reported its anti-inflammatory and immunosuppressive effects in chronic urticaria (whether autoimmune or not). ${ }^{4,5}$ MTX is a relatively safe drug for patients with chronic urticaria. Gastrointestinal discomfort and a mild increase in liver enzymes are adverse effect of MTX treatment that can be resolved with intramuscular administration and reduced dosage, respectively. $^{2}$

Pneumocystis jirovecii pneumonia has been reported in patients with rheumatoid and psoriatic arthritis, Behcet disease, periarteritis nodosa, and malignancy of the larynx who are receiving MTX therapy. ${ }^{6-7}$ Low CD4+ lymphocyte counts in patients receiving MTX therapy and without HIV infection have been proposed as a risk factor for Pneumocystis jirovecii pneumonia infection. ${ }^{6}$ The total lymphocyte count in our patient was 256 cells/mL at the time of diagnosis of Pneumocystis jirovecii pneumonia, which supports this idea, even without lymphocyte panel analysis (normal CD4+ lymphocyte count, 550-1190/mL).

Kane et al. suggested that patients receiving cumulative MTX doses exceeding $400 \mathrm{mg}$ should be monitored for the development of Pneumocystis jirovecii pneumonia. ${ }^{6}$ The total dosage of MTX used in our patient was $195 \mathrm{mg}$. The development of infection may be attributed to the concomitant use of low-dose systemic corticosteroids for $\sim 1$ year. A reduction in the CD4+ lymphocyte count and impaired cell-mediated immune activity are the vital effects of corticosteroid use that increase the predisposition to infection with Pneumocystis jirovecii pneumonia. ${ }^{8}$

The typical chest radiography feature of Pneumocystis jirovecii pneumonia is bilateral reticular interstitial pattern with perihilar distribution. However, $\sim 10 \%$ to $15 \%$ of patients who acquire Pneumocystis jirovecii pneumonia have normal chest radiographic findings. ${ }^{9}$ High-resolution computed tomography, which is more sensitive than chest radiography, is a good diagnostic tool in patients with a typical presentation of Pneumocystis jirovecii pneumonia such as progressive dyspnea, nonproductive cough, and lowgrade fever but normal chest radiographic findings. ${ }^{10}$

In conclusion, Pneumocystis jirovecii pneumonia should be considered in the differential diagnosis of patients with refractory chronic urticaria treated with weekly MTX if dyspnea, cough, and fever develop, especially in those with a history of concomitant use of systemic corticosteroids or a low $\mathrm{CD} 4^{+}$lymphocyte count. The efficacy and safety of MTX and the corticosteroid dose-tapering strategy used after MTX therapy need to be assessed in randomized control trials. $\square$ 


\section{REFERENCES}

1. Asero R, Tedeschi A, Cugno M. Treatment of refractory chronic urticaria: current and future therapeutic options. Am J Clin Dermatol. 2013;14:481-8.

2. Sagi L, Solomon M, Baum S, Lyakhovitsky A, Trau H, Barzilai A. Evidence for methotrexate as a useful treatment for steroid-dependent chronic urticaria. Acta Derm Venereol. 2011:91:303-6.

3. Criado PR, Criado RF, Maruta CW, Reis VM. Chronic urticaria in adults: state-ofthe-art in the new millennium. An Bras Dermatol. 2015;90:74-89.

4. Gach JE. Methotrexate-responsive chronic idiopathic urticaria: a report of two cases. Br J Dermatol. 2001;145:340-3.

5. Perez A, Woods A, Grattan CE. Methotrexate: a useful steroid-sparing agent in recalcitrant chronic urticaria. Br J Dermatol. 2010;162:191-4.

6. Kane GC. Pneumocystis carinii pneumonia associated with weekly methotrexate: cumulative dose of methotrexate and low CD4 cell count may predict this complication. Respir Med. 1993;87:153-5.

7. Yoshida $\mathrm{Y}$, Takahashi $\mathrm{Y}$, Minemura $\mathrm{N}$, Ueda $\mathrm{Y}$, Yamashita $\mathrm{H}$, Kaneko $\mathrm{H}$, et al. Prognosis of pneumocystis pneumonia complicated in patients with rheumatoid arthritis (RA) and non-RA rheumatic diseases. Mod Rheumatol. 2012;22:509-14.

8. Tabarsi P. Inappropriate use of steroid and pneumocystis jiroveci pneumonia: report of two cases. East Mediterr Health J. 2008;14:1217-21.

9. Opravil M, Marincek B, Fuchs WA, Weber R, Speich R, Battegay M, et al. Shortcomings of chest radiography in detecting Pneumocystis carinii pneumonia. J Acquir Immune Defic Syndr. 1994;7:39-45.

10. Thomas CF Jr, Limper AH. Pneumocystis pneumonia. N Engl J Med. 2004;350:2487-98
MAILING ADDRESS:

Sheng-Huei Wang

No. 325, Section 2

Cheng-Kung Road

Neihu 114,

Taipei, Taiwan, ROC.

E-mail: slidersinker@gmail.com

How to cite this article: Wang SH, Tang SE, Li YH, Wei KY, Chang CY. Pneumocystis jirovecii pneumonia induced by low-dose methotrexate in a patient with chronic urticaria. An Bras Dermatol. 2017;92(3):401-3. 